Document downloaded from:

http://hdl.handle.net/10251/45113

This paper must be cited as:

Ghafoor, KZ.; Abu Bakar, K.; Lloret, J.; Khokhar, RH.; Lee, KC. (2013). Intelligent beaconless geographical forwarding for urban vehicular environments. Wireless Networks. 19(3):345-362. doi:10.1007/s11276-012-0470-z.

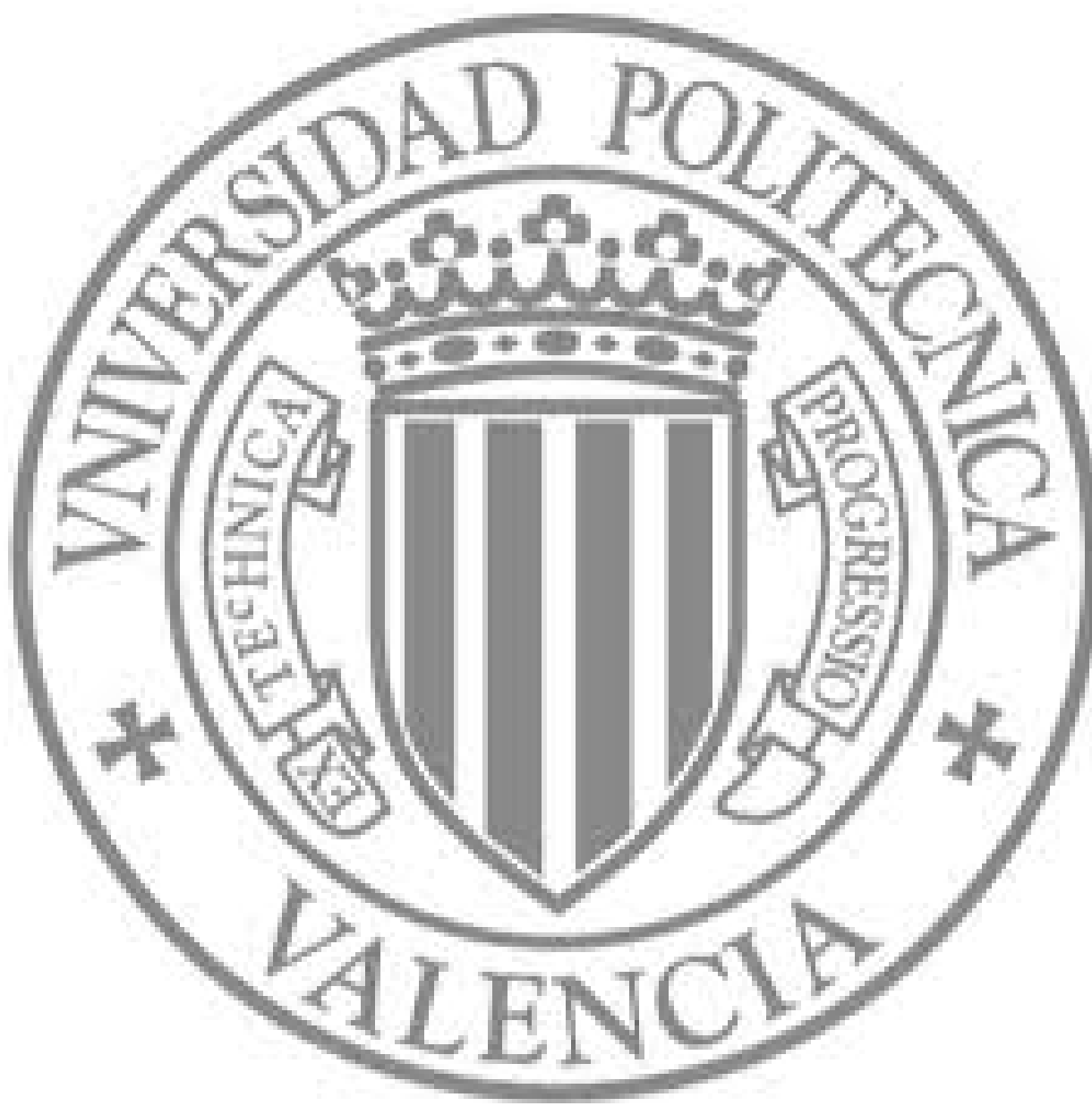

The final publication is available at

http://dx.doi.org/10.1007/s11276-012-0470-z

Copyright Springer Verlag (Germany) 


\title{
Intelligent Beaconless Geographical Forwarding for Urban Vehicular Environments
}

\author{
Kayhan Zrar Ghafoor · Kamalrulnizam Abu Bakar \\ - Jaime Lloret · Rashid Hafeez Khokhar - Kevin C. Lee
}

the date of receipt and acceptance should be inserted later

\begin{abstract}
A Vehicular Ad hoc Network (VANET) is a type of wireless ad hoc network that facilitates ubiquitous connectivity between vehicles in the absence of fixed infrastructure. Source based geographical routing has been proven to perform well in unstable vehicular networks. However, these routing protocols leverage beacon messages to update the positional information of all direct neighbour nodes. As a result, high channel congestion or problems with outdated neighbour lists may occur. To this end, we propose a streetaware, Intelligent Beaconless (IB) geographical forwarding protocol based on modified 802.11 Request To Send (RTS)/ Clear To Send (CTS) frames, for urban vehicular networks. That is, at the intersection, each candidate junction node leverage digital road maps as well as distance to destination, power signal strength of the RTS frame and direction routing metrics to determine if it should elect itself as a next relay node. For packet forwarding between Intersections, on the other hand, the candidate node considers the relative direction to the packet carrier node and power signal strength of the RTS frame as routing metrics to elect itself based on
\end{abstract}

Kayhan Zrar Ghafoor · Kamalrulnizam Abu Bakar

Faculty of Computer Science and Information Systems, Universiti Teknologi Malaysia,

81310 UTM Skudai, Johor D. T, Malaysia.

Tel.: +6-017-7193871

E-mail: kayhan@ieee.org

Jaime Lloret

Departamento de Comunicaciones, Universidad Politecnica de Valencia Camino de Vera s/n, 46022, Valencia, Spain.

Rashid Hafeez Khokhar

Faculty of Computer Science and Information Technology, University of Malaya, 50603 Lembah Pantai, Kuala Lumpur, Malaysia.

Kevin C. Lee

Department of Computer Science, University of California, Los Angeles, CA 90095, USA. intelligently combined metrics. After designing the IB protocol, we implemented it and compared it with standard protocols. The simulation results show that the proposed protocol can improve average delay and successful packet delivery ratio in realistic wireless channel conditions and urban vehicular scenarios.

Keywords VANET - multi-metric based self election . IEEE $802.11 \cdot$ street map aware routing

\section{Introduction}

The recent growth of wireless communication technologies paves the way for many emerging mobile networks like VAN ET. This type of network facilitates the communication between vehicles in the absence of fixed infrastructure. Thus, the increasing necessity of this network is an impetus for leading car manufacturers, research communities and governments to increase their efforts toward creating a standardized platform for vehicular communications. In particular, the $5.9 \mathrm{GHz}$ spectrum band has been allocated for licensed Short Range Communication (DSRC) between vehicles. Moreover, in the near future, vehicles will be equipped with wireless devices such as Wireless Access in Vehicular Environment (WAVE) [?]. With this communication capability, vehicles can exchange information, enabling numerous applications and services that are unique to vehicular traffic scenarios. The applications include traffic safety, cooperative traffic monitoring and control of traffic flow. These applications are made possible by the use of efficient multihop routing protocols, which are a significant departure from short range communications to the wide coverage areas coverage. Clearly, in VANET, this long distance communication can be achieved using multi-hop routing approaches. Various type of routing protocols and their shortcomings are extensively discussed in the next section. 
The rest of the paper is arranged as follows: section 2 discusses the recent related literature. Section 3 provides an overview of 802.11 RTS/CTS protocol. In section 4, we discuss an overview of the proposed protocol. The algorithm of the proposed protocol is discussed in section 5. This is followed by developing the score functions for relay self election at and between intersections in section 6 . In section 7, performance evaluation is elaborated, where we highlight the feasibility of our protocol by considering a realistic urban vehicular scenario and log-normal shadowing model. Finally, section 8 concludes the paper.

\section{Related Work}

\subsection{Geographical Routing Protocol}

VANET is counted as a special type of Mobile Ad hoc Networks (MANET) where vehicles or transportation infrastructures equipped with wireless access to form a self organizing wireless network. Traditional routing protocols designed for MANET, Ad hoc On Demand Distance Vector (AODV) [?]; Dynamic Source Routing (DSR) [?], are less preferable in vehicular environments. These protocols are address based rather than position based i.e., discover and maintain the end-to-end path between source and destination. This leads to frequent break down of the routes due to unstable vehicular networks. As a result, the protocol suffers from control overhead, leading to low network performance. Fig. ?? shows the problem of address based routing in the urban vehicular environment. As can be seen, the established route between the source and the destination breaks.

An alternative routing scheme, which is suitable for vehicular environments, is geographical routing where vehicles' route data packets by considering the position of the

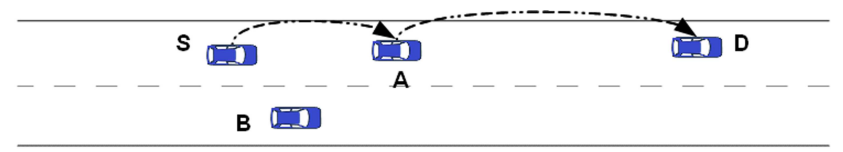

(a) At time $\mathrm{t}=t_{1}$.

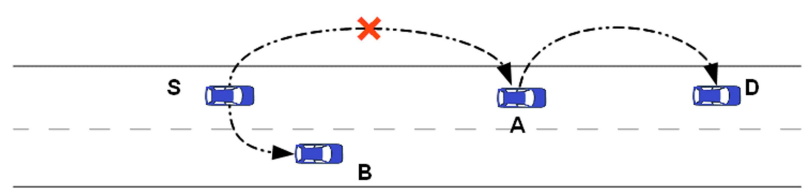

(b) At time $\mathrm{t}=t_{1}+\delta t$.

Fig. 1: The address based routes that were established between the source and the destination frequently break due to unstable vehicular networks. Route $(S, A, D)$ that was created at the time $\mathrm{t}=t_{1}$, breaks at the time $t=t_{1}+\delta t$ when $A$ exit the radio range of $\mathrm{S}$. destination node. This type of routing is more desirable in VANET for the following reasons. First, in the near future, vehicles will be embedded with Global Positioning System (GPS) and navigation systems, hence geographical routing is perfectly suited to VANET. Second, since geographical routing is stateless, it does not maintain established routes between the source and the destination.

In vehicular wireless networks, the performance of existing geographical routing protocols, such as [?], [?], [?], [?], [?], [?], [?], [?], [?] has been improved by considering efficient forwarding strategies and vehicular mobility characteristics. However, each of the aforementioned routing protocols has its own limitations. Greedy Position Stateless Routing (GPSR) [?] may not perform well because of uneven traffic distribution- a combination of dense and sparse traffic conditions- along different road segments. Under these circumstances, GPSR activates face routing, in which a data packet is forwarded on a series of faces towards the destination. Greedy Source Routing GSR [?], uses greedy relaying to forward data packets toward the destination. However, they disregard low traffic densities in which there are an insufficient number of nodes to relay data packets. Mobilitycentric data dissemination (MDDV) protocol [?] considers the concepts of trajectory and opportunistic forwarding to transmit messages towards destination regions. However, as traffic density changes with time, MDDV enters local optimum with large amounts of route latency. Some authors in [?] developed Anchor-based Street and Traffic Aware Routing (A-STAR) to utilize bus route information to discover the best anchor paths of higher connectivity toward the packet's destination.

Based on the assumption that road traffic is the planar graph, the authors in [?] proposed Greedy Perimeter Coordinator Routing (GPCR) which utilizes the concept of junction nodes to control the next road segments that packets should follow. In this new type of planar graph based on underlying roads, vehicles can forward data packets along the streets in both greedy and perimeter modes. At the intersection, GPCR uses two heuristics to determine whether a node is located at the intersection. The first one uses beaconing services so that each node is aware about its neighbours. A node can be considered a coordinator node when it has two neighbours that are within radio range of each other, but do not list each other as a neighbour. The second one is derived by calculating the correlation coefficient that relates a node to its neighbours. A correlation coefficient close to zero indicates that there is no linear coherence between the positions of the neighbours. This indicates the node is located at a junction. GPCR solves the problem of inaccuracy of node popularization in addition to the improvement of forwarding performance as packets travel less number of nodes in the perimeter mode. Performance evaluation shows that GPCR delivers more data packets compared to GPSR. However, 
there is the possibility that packets loop back in the same street from which the packet has arrived. Furthermore, they only considered greedy forwarding metric for packet routing in error prone urban vehicular scenario.

The authors in [?] proposed diagonal-intersection-based routing (DIR) protocol for routing packets in urban vehicular scenario. The DIR protocol consists of three phasesdestination discovery, packet forwarding, and route maintenance, to route packets efficiently towards the destination. However, the periodic maintenance of link cost (expected packet forwarding delay) between forwarding diagonal intersections (from $I_{x i, y i}$ to $I_{x j, y j}$ ) leads to higher overhead traffic and hence negatively creates an impact on the endto-end data transfers.

Another direction of routing data packets optimally over urban vehicular environments is taken by Jerbi et al. in [?] where the authors proposed an improved vehicular ad hoc routing protocol for city environments (GyTAR). The designed protocol has two modes of operation: routing at the intersections and at road segments. For the former mode, GyTAR reactively selects the neighbour intersections upon consideration of variations in traffic density and the distance to the destination. However, since realistic city maps have irregular shapes such as unequal road segments between intersections, GyTAR does not consider variations of segment lengths within urban environments. Furthermore, GyTAR's traffic density estimation is very costly in terms of bandwidth consumption and scalability issue.

Cheng et al. discusses that delay tolerant aware routing protocol is necessary due to heterogeneous distribution of vehicles. To achieve such packet salvaging capability, in [?] the authors proposed GeoDTN+Nav in which they combined greedy mode, perimeter mode, and DTN mode. The GeoDTN + Nav protocol utilizes a network partition detection method so as to switch between different modes of packet forwarding. Network partition detection switches between different modes based on the number of hops a packet has travelled so far and the delivery quality of neighbours. The Virtual Navigation Interface (VNI) has been used to provide necessary information for the proposed protocol so that it can determine its routing mode and next hop forwarder. However, the developed protocol have shortcomings in terms of favouring link reliability, stability and forwarding progress toward destination. In [?], the authors developed address based geoopportunistic routing that uses topology assisted geographic routing with opportunistic forwarding. The proposed routing protocol uses periodic packet receptions evoked by broadcast wireless medium and forwarding opportunity is triggered to perform packet routing through direct neighbours that have received data packets successfully.

In another attempt, Kayhan et al. in [?] developed a Stability and Reliability aware routing protocol (SRR) for vehicular networks. The SRR protocol incorporates fuzzy logic with geographical routing in order to lend cognitive capability to packet forwarding decisions. Routing metrics, including direction and distance, are considered inputs of the fuzzy decision making system in order to select the best preferable route around a smart vehicle. Next, it proposes a local decision mechanism to observe the network partitions in order to allow switching from SRR mode to queuing mode or vice versa. In contrast to the proposed IB protocol, the SRR routing protocol uses fuzzy inference system to rank the neighbour nodes and considers source based routing for relay node selection. In addition, the SRR protocol is designed for highway vehicular scenario rather than urban vehicular network.

\subsection{Beaconless Geographical Forwarding}

Neighbour discovery is a crucial part of geographical routing protocols. To achieve this, the routing protocols assume that nodes broadcast periodic beacon messages to inform neighbour nodes about their address, location and other relevant information. In this proactive neighbourhood awareness, each vehicle must maintain an up-to-date list of neighbour nodes. Otherwise, the outdated information problems occur, where of the neighbour list leads to a miss of the next candidate node, or the node that has been chosen will move out the radio range (Fig. ??).

The authors in [?] proposed a geographical forwarding scheme called Guaranteed Delivery Beaconless Forwarding Scheme (GDBF). In the proposed scheme, the relay node is selected through the use of control RTS/CTS frames of the MAC layer and waiting time function. In greedy mode, the candidate node which is closest to the destination responds to the source first.

When a source node has shortest distance to the destination as compared to the distance of direct neighbour nodes, the contention winner might be the node which is closer to the source. Thus, other nodes which overhear the CTS frame exit from the contention phase because there is a link established with the source.The GDBF could guarantee packet delivery as compared with the existing beaconless routing protocols. Furthermore, the existing beaconless approaches either retransmit the whole data packet immediately, that might lead to the redundant retransmissions, or have duplicate packets.

In their analysis, they confirmed low routing overhead and high guaranteed delivery. However, they assumed ideal MAC layer and unit disc radio propagation in their performance evaluation. Since the wireless channels between vehicles in the urban environment are error prone due to high inter-channel variation, shadowing and fading effects, the aforementioned authors did not consider the quality of wireless channel and stability of packet forwarding. In addition, 


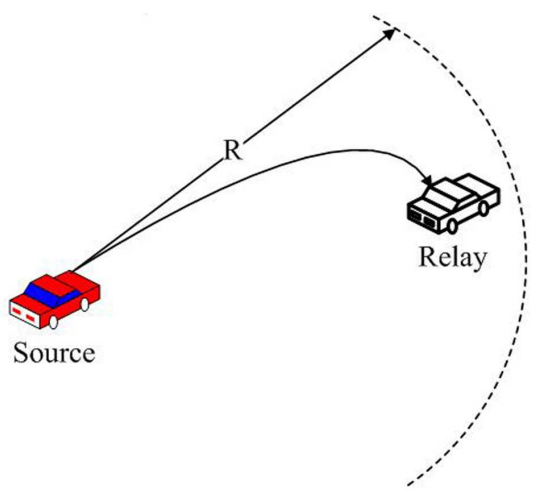

(a) The source node handshakes,through beaconing, with the relay node before sending data packets.

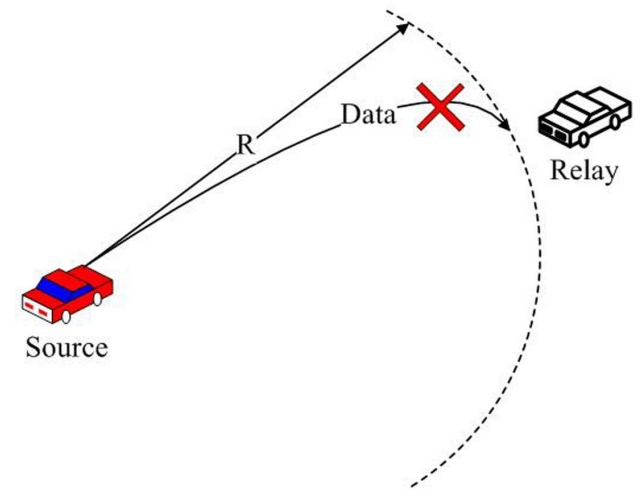

(b) The relay node exit the radio range after sending data packets by the source.

Fig. 2: Inaccurate neighbour list problem in VANET.

their proposed scheme is not designed for urban vehicular environments.

In [?], Fussler et al. proposed a Contention Based Forwarding (CBF) to route data packets greedily without the need of periodic beacon transmissions. The packet carrier node, which runs CBF, does not score the direct neighbour nodes. Rather, it broadcasts the control frames to them, and they should decide individually whether to forward a packet or not. That is, the packet carrier node broadcasts the RTS frame containing its and the destination position. Then the next relay node is selected by distributed timer-based next hop self election in the contention period. The winner (shortest reply time) of the contention phase is the node which has more geographical progress toward the destination. The contention winner broadcasts the CTS frame to the node, which is an originator of the RTS frame. At this time, the candidate nodes that hear this CTS frame, cancel their timers and exit from the contention process. The CBF protocol did not consider the unreliability and instability issues of packet forwarding by considering other parameters like power signal or direction of movement. Thus, this protocol may lead to sub-optimal results in erasure wireless channel.

A positive step toward efficient routing protocols is taken by [?], where the authors proposed road-based routing protocols that leverage on-board navigation systems to establish paths between the source and the destination through a sequence of intersections with high network connectivity. In addition, to eliminate the hello packet, the authors proposed an enhancement of receiver-based next hop self election (which is the core of our proposed protocol) (e.g., [?] or [?]) to reduce protocol overhead in the network. However, exchanging link state information and route maintenance leads to high network overhead. In [?], the authors use beaconless forwarding optimization between intersections; however, it does not consider packet forwarding decision at intersections. In addition, they focused on the power signal strength, optimal transmission range and distance for packet forwarding. On the contrary, we take into account direction, forwarding progress and variation of received signal strength for reliable, stable and fast packet forwarding. Furthermore, the modification of RTS/CTS in IB protocol is far different compared with their geographical forwarding.

In [?], Ruhrup et al. proposed beaconless georouting with guaranteed delivery for wireless sensor, ad hoc and actuator networks. The proposed protocol, which is based on the Select-and-Protest principle, consists of two methods for reactive face routing with guaranteed delivery. The Beaconless Forwarder Planarization (BFP) determines the nodes of a local planar subgraph with using beacon message form all neighbours. The second method uses angular relaying to determine next hop of a righthand face traversal. In addition, the authors presented Circlunar Neighbourhood Graph (CNG) to address the planarization problems of Gabriel Graph (GG). More precisely, less messages is needed to construct CNG than GG. Simulation and theoretical studies show that the proposed delay function reduced the number of protest messages by a factor of 2 as compared to the angle-based delay function.

In the brief discussion above, it is clear that receiver based self election is an imperative need for multi-hop routing along the streets in the urban vehicular environment. To this end, in this paper, we propose Intelligent Beaconless (IB) geographical forwarding protocol, based on modified 802.11 RTS/CTS frames, for vehicular wireless networks. That is, at the intersection (which is defined as a convergence of one or more Road Segments), each candidate junction node leverage digital road maps as well as distance to the destination, power signal strength and direction to the next coming intersection routing metrics to determine if it should elect itself as a next relay node. For packet forwarding between Intersections, on the other hand, 
the candidate node considers the relative direction to the packet carrier node and power signal strength of the RTS frame as routing metrics to elect itself based on intelligently combined metrics. The proposed geographical forwarding protocol has been modeled using JIST/SWANs [?] network simulator tool for performance evaluation. It is noteworthy that the IB protocol is well suited for many applications. For example, in comfort-related applications, it can be used for chatting, gaming or infotainment between vehicles. We summarize the contributions of this study as follows:

- We propose IB geographical forwarding protocol to tackle proactive neighbour discovery. In essence, for packet forwarding at the intersection, the candidate junction nodes utilize distance to the destination, power signal strength and direction as routing metrics. For routing between intersections, multi-metric packet forwarding has also been utilized to forward data packets toward the destination.

- We perform simulations to show the effect of vehicular traffic density and speed of vehicles on the proposed geographical forwarding protocol.

\section{An Overview of 802.11 RTS/CTS protocol}

The IEEE 802.11 Distributed Coordination Function (DCF) [?], [?] is designed to implement the Carrier Sense Multiple Access/Collision Avoidance (CSMA/CA) protocol, which utilizes four way handshaking (RTS/CTS/Data/ACK) for a session of data transmission. When the source node has data for transmission, it senses the wireless channel for the period called DCF Inter Frame Space (DIFS). If the channel is idle for DIFS period, then it selects a random backoff timer in the range $(0, \mathrm{CW})$, where $\mathrm{CW}$ is Contention Window. After the timer expires, the source sends the RTS frame to the intended receiver. The receiver broadcasts the CTS frame to all of its neighbours. The neighbours of the source and the intended receiver update their Network Allocation Vector (NAV) in the duration during which the channel is occupied by the ongoing session. During this time interval, all neighbours defer their transmission until this session is completed. After the source has received the CTS frame, it starts sending data to the intended receiver, and this is followed by an ACK frame if the data is received successfully. In case of any failure of data transmission the source starts the retransmission until retry limit is reached.

\section{Proposed Protocol Overview}

The designed IB protocol is adopted for Inter-Vehicle Communication (IVC), in which vehicles communicate in the absence of fixed infrastructure. The proposed protocol considers that all vehicles are equipped with the Global Positioning System (GPS) services and digital road map, i.e. each vehicle knows its own position, coordinates of the junctions and road segments. This assumption is valid because in the near future more and more vehicles will be equipped with on-board navigation systems. The packet carrier node (source/relay nodes) needs to have the position coordinates of the destination node to make packet forwarding decisions. Furthermore, the proposed protocol is simulated in an open and regularly structured vehicular environment (i.e., radio obstacles and dead-end roads are not considered in the simulation).

In addition, the IB protocol does not require the transmission of beacon messages and forward data packets optimally in urban vehicular networks. To achieve this objective, it makes the packet forwarding decision at and between intersections. Fig. ?? demonstrates the flowchart steps of the of IB protocol. The dark gray blocks are the contributions of the designed IB protocol. In essence, at the intersection the packet carrier node broadcasts a modified control frame called RTS to all direct neighbours and the neighbours themselves decide if they should forward data packets. This forwarding decision lies on the distributed timer-based contention process, which allows the most suitable candidate node to access the channel based on the distance to the destination, power signal strength and the relative direction to the next coming intersection. After the best intersection has been chosen, and while between intersections, the candidate nodes try to access the channel based on multi-metric forwarding decision, i.e. the power signal strength of the RTS frame as well as relative direction between the candidate and the packet carrier node. When the best node, whether at or between intersections, accesses the channel, the remaining nodes then cancel the scheduled packet after detecting this transmission. In the next section, the complete algorithm of IB protocol is discussed.

\section{Proposed Protocol Algorithm}

IB protocol is completely unlike source based routing, which is based on receiver self election to suppress the effect of frequent broadcast of beacon message. The distributed next hop self election is based on modified RTS/CTS frames of the 802.11 protocol. The general contention phase procedure is demonstrated in Fig. ??. As can be seen, if the packet carrier node receives a single CTS frame, then it transmits (after Short Inter-Frame Space (SIFS)) the data frames to the node which wins the contention phase. Furthermore, the design of the score function, which is used to compute the reply timer, has an important role in the successful contention phase between RTS receiver nodes. 


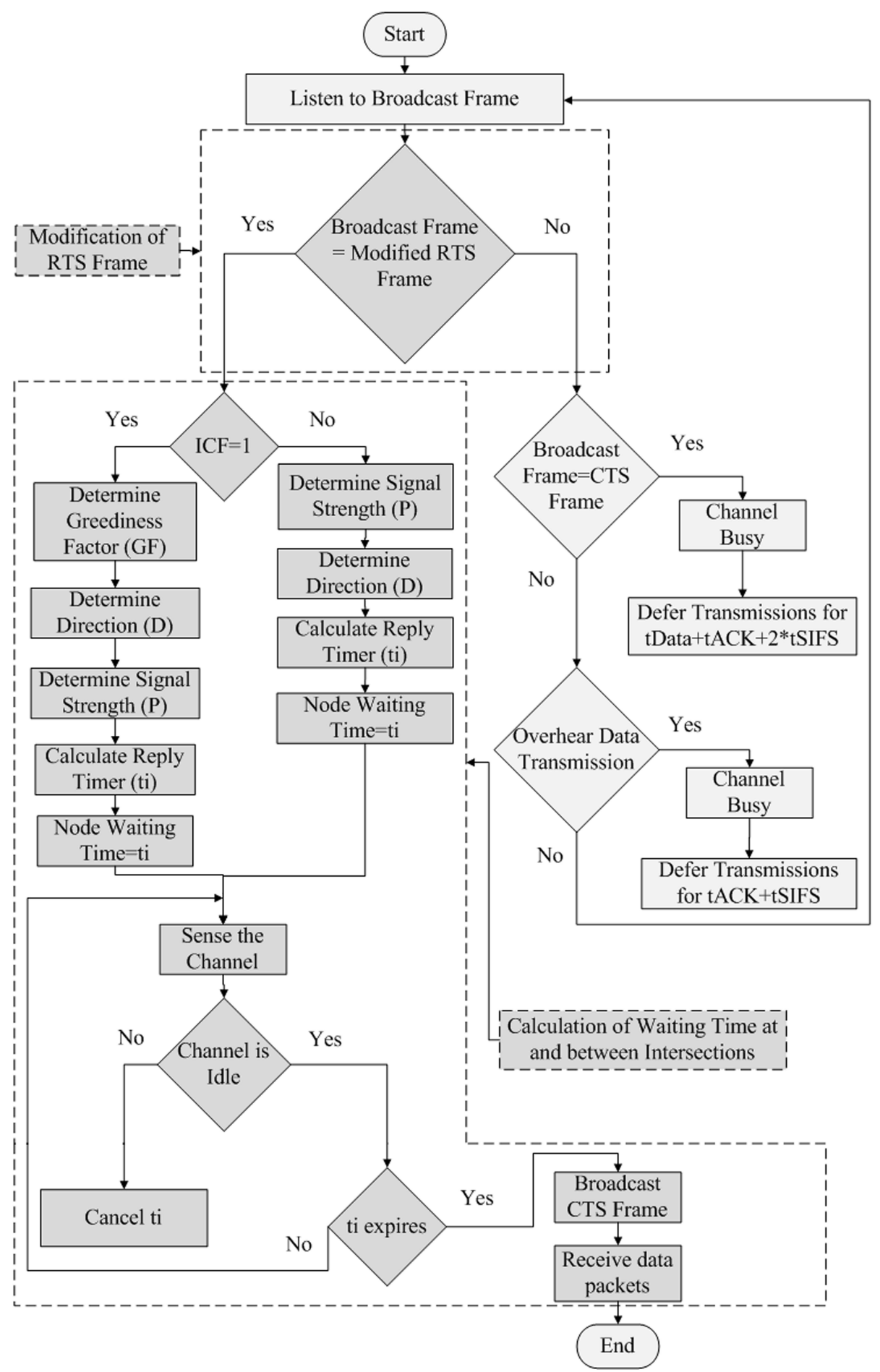

Fig. 3: Flowchart of different steps for IB geographical forwarding protocol

In addition, the RTS is a broadcast message which is modified to carry the position of the destination node, the direction and position of the source node. Furthermore, it also carries two flags: the first one is used by the receivers to know whether the source is located at the intersection or between them, and the second flag is useful to make all receiver nodes to process/respond the RTS broadcast message.

The details of the IB protocol are illustrated in Algorithm 1. From line 9 to 10 , upon receiving the RTS frame, candidate nodes first check, through Intersection Check Flag (ICF), whether or not the source is at the intersection or be- 


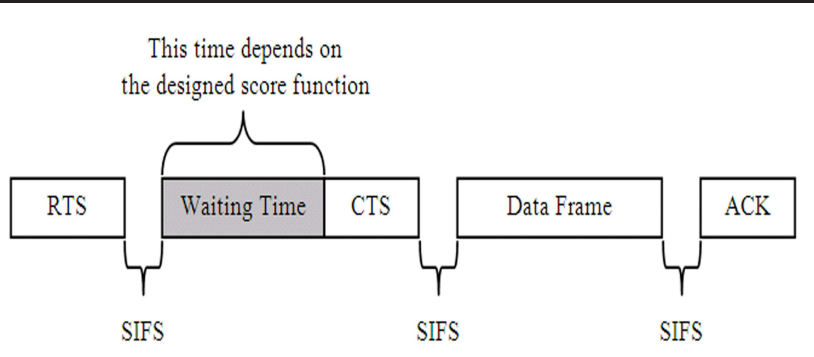

Fig. 4: Contention phase of the IB protocol

tween them (line 10). At the intersection $(\mathrm{ICF}=1)$, a higher priority should be given to a node, which provides forward progress, loop-free for the packet proclaimed by the RTS frame, and strong signal strength of the RTS frame. Therefore, candidate nodes compute their score (reply timer) based on Greediness Factor, Direction, and Signal Strength (line 11-13). After determining the reply timer $t_{i}$, candidate nodes set their timer to the value of $t_{i}$, and this value determines how good the candidate node is to become a next packet forwarder (line 14-16).

If the current packet carrier node is located between intersections $(\mathrm{ICF}=0)$ (line 17), the candidate node measures the signal power strength (Signal Strength) of the RTS frame and computes its direction (Direction) relative to the packet carrier node. Then, the candidate nodes compute their reply timer. This reply timer depends upon the relative direction and power signal strength: a candidate node has a shortest reply timer when it travels in the same direction of the packet carrier node as well as receives the RTS message with a strong power signal (line 18-23).

In case of the reply timer beetle off, a control frame called CTS is transmitted to the packet carrier node, which indicates that it wins the contention phase and becomes the best preferable relay node. Meanwhile, when the direct neighbour nodes hear a CTS frame, they cancel their own timer and are suppressed from the contention phase. Afterwards, the source concludes the communication session by transmitting data packets to the elected node (line 24-34). It is noteworthy to mention that if the reply timer $t_{i}$ has infinite or negative values, the packet will be discarded by the candidate node. In the next section, the score function for relay self election at intersections is illustrated.

\section{Proposed Forwarding Metrics}

\subsection{Score Function for Relay Self Election at the} Intersections

In multi-hop routing protocols, packet forwarding based on one routing metric can be sub-optimal because many criteria (reliability, stability, one hop progress) affect route optimization between the source and the destination. At the

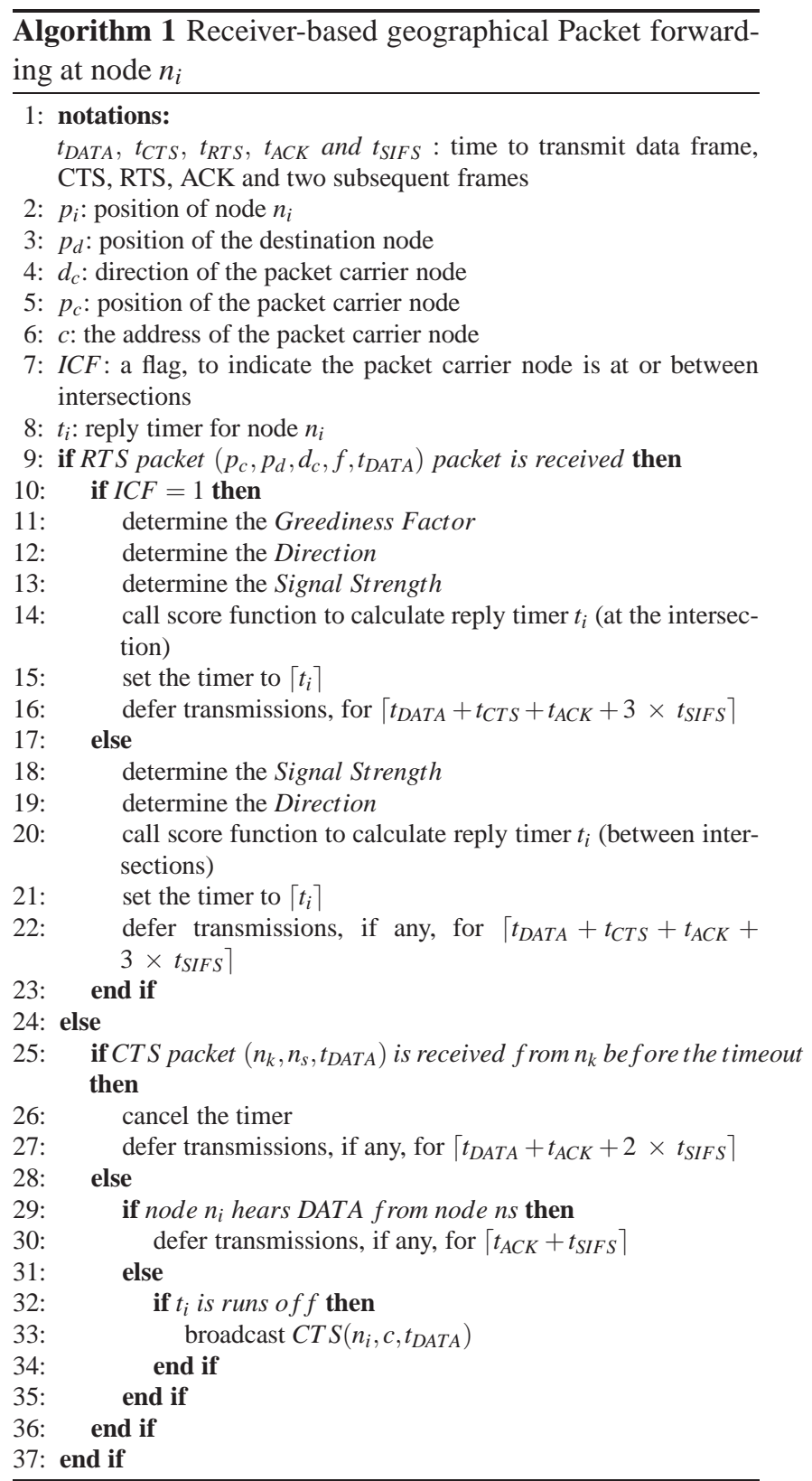

intersections, the best preferable relay node is determined based on the reply timer. The reply timer is computed based on the (multi-metric) score function. Thus, to qualify the best preferable candidate node, we introduce the input parameters of the score function: distance to the destination, direction towards the next coming intersection and power signal strength of the RTS frame.

1) Greediness - Factor $(G F)$ : When a candidate junction node is at the intersection and receives the RTS frame, it calculates the Greediness - Factor which indicates the closeness of a candidate node $n_{i}$ to the destination. This factor is calculated by $D_{c} / D_{i}$; where $D_{i}$ is the distance of a node $n_{i}$ to the destination and $D_{c}$ is the distance of the current packet carrier node to the destination. The greater the 
Greediness - Factor is, the higher priority a node has, and hence it is approaching the destination with high advancement progress. We considered the Greediness - Factor routing metric due to crucial decision of packet routing at the intersection. However, if we consider this parameter alone, the routing loop might be occurring at the intersection. Thus, we consider the direction of a candidate node toward the next coming intersection.

2) Direction $(D)$ : The direction of vehicles is important to be considered for stable packet forwarding. This is because the direction of vehicles is constrained by the roads as well as two vehicles travelling in the same direction having more stable wireless link than they travel in the opposite direction. In realistic urban scenarios (road with curvature), the direction vector of vehicles is not always paralleling to each other. However, the proposed protocol considers straight roads in the urban environments i.e. vehicles are travelling in the same or opposite directions. Thus, we place a great deal of reliance on this feature to give higher priority to the nodes that they travel to the next coming intersection. The relative direction between a mobile node and the fixed point is calculated by measuring the angle between direction vector of the mobile node and the x-axis [?]. Furthermore, since wireless channel between vehicles is error prone, the channel quality is also should be considered in packet forwarding decisions.

3) Signal-Strength $(P)$ : The wireless channels between vehicles are susceptible to attenuation and fading due to buildings and other obstacles in the urban area. Therefore, we consider the level of power signal as a metric to characterize the quality of channel between a candidate node and the packet carrier node. A candidate node can determine this parameter by measuring the power signal level of the RTS frame.

After the routing metrics has been defined, an aggregating function should be used to combine all criteria into a single function which is used to excel the best candidate node. The score function is a single ranking measure that combines all routing metrics into a single one. Since the random backoff timer of IEEE 802.11 is based on product of slot time and a random number, the score function is also modelled as a multiplication of specified variables. Consider a score function based on $j$ routing metrics $\zeta_{i}=$ $\left\{\zeta_{i 1}, \zeta_{i 2}, \zeta_{i 3}, \ldots, \zeta_{i j}\right\}$ (In this paper, we assume that the routing metrics has to be maximized), for each of them the candidate node $n_{i}$ has numerical values in the range $\left[\zeta_{i}^{\min }, \zeta_{i}^{\max }\right]$. Then, a multi-metric scoring function is given as follows [?]:

$f\left(\zeta_{i 1}, \zeta_{i 2}, \ldots, \zeta_{i j}\right)=X \times \zeta_{i 1}^{\beta_{1}} \times \zeta_{i 2}^{\beta_{2}} \times \zeta_{i 3}^{\beta_{3}} \ldots . \zeta_{i j}^{\beta_{j}}+Y_{\max }$

where $Y$ is the maximum value of the scoring function $f\left(\zeta_{i 1}, \zeta_{i 2}, \zeta_{i 3}, \ldots, \zeta_{i j}\right), X$ is the variable dependent weights of the limiting condition, and $\left(\beta_{1}, \beta_{2}, \beta_{3} \ldots, \beta_{j}\right)$ is a $\mathrm{j}$-weight array used to give priority to the routing decision metrics, i.e. the routing metric with a higher weight factor has more impact on the self election process. In our IB protocol, three metrics has been utilized to make packet forwarding decision at the intersections. Thus, the reply timer value is calculated as follows:

$$
f\left(G F_{i}, D_{i}, P_{i}\right)=X \times G F_{i}^{\beta_{1}} \times D_{i}^{\beta_{2}} \times P_{i}^{\beta_{3}}+Y_{\max }
$$

The maximum value of $f\left(G F_{i}, D_{i}, P_{i}\right)$ occurs when its derivative equals zero, the value of $\mathrm{X}$ is given by:

$X=\frac{-Y_{\text {max }}}{G F_{\text {max }}^{\beta_{1}} \times D_{\text {max }}^{\beta_{2}} \times P_{\text {max }}^{\beta_{3}}}$

To evaluate the score function in equation ??, first we need to know the maximum values of $G F_{i}, D_{i}$ and $P_{i}$. The maximum value of $G F_{i}$ depends on the simulation area and the communication range of vehicles. Accordingly, the $G F_{\max }$ is equal to (10). The maximum value of $D_{i}$ is (1). This is because the maximum range of $\cos \sigma$ is 1 ( $\sigma$ is the angle between direction vector of the candidate node and $\mathrm{x}$-axis).

Furthermore, we conducted a real experiment to determine the maximum value of the signal power. We set up a scenario whereby two vehicles equipped with laptops are travelling in the same direction in urban vehicular environment. The computer laptops have 802.11b Wireless LAN card as well as software for signal strength measurement named "InSSIDer 2" [?]. Furthermore, during the experiment, both vehicles travelled with an average speed of 45 $\mathrm{km} /$ hour. Therefore, based on this experiment, we observe the maximum value of $P$ is $(-20 \mathrm{dBm})$ and the minimum value is $(-60 \mathrm{dBm})$. The $(-60 \mathrm{dBm})$ is the radio reception threshold and below this value the data packet receptions are not possible.

If the maximum time delay $\left(Y_{\max }\right)$ for a candidate node election is $0.8 \mathrm{~ms}$ (this value empirically is set in the simulator), $\beta_{1}=0.5, \beta_{2}=0.2, \beta_{3}=0.01$ and then we use equation ?? to calculate the value of $X$ which yields -0.2416 . Therefore, we can compute the reply timer $\left(t_{i}\right)$, based on equation ??, which is equal to $0.0860 \mathrm{~ms}$. This duration (0.08

$60 \mathrm{~ms}$ ) is the time in which the candidate node should wait after receiving the RTS message. It is noteworthy that all candidate nodes leverage the score function (equation ??) to compute their reply timer after receiving the RTS message. Moreover, for theoretical analysis the values of weights are constant, but they optimally could be determined in the performance evaluation.

Furthermore, Fig. ?? depicts the correlation behaviour between $t_{i}, G F_{i}$ and $P_{i}$ variables. The trend shows that the reply timer $\left(t_{i}\right)$ decreases to minimum value when the value of $P$ is $0.01 \mathrm{mw}$ and $G F$ is 10 . This is because the candidate 
node is more directed toward the next coming intersection, is close to the destination node and is receive the the RTS frame with strong power signal (lower green part).

The IB protocol handles two modes of packet forwarding in the urban vehicular environment. In the previous sections, we presented the packet forwarding decision at intersections. In the next section, we elaborate the routing metrics and the score function for packet forwarding between intersections.

\subsection{Score Function for Relay Self Election between Intersections}

In this section, we use the same ideas of equation ?? to derive a multi-metric/score function for packet forwarding between intersections. This score function consists of two input variables and one output variable. The inputs are the power signal strength of the RTS frame and relative direction of a candidate node with respect to the packet carrier node. The power signal strength of the RTS frame is discussed in section ??, the second metric is the the movement direction of the vehicles Direction $(D)$, which facilitate stable packet forwarding towards the destination. This is because the direction of vehicles is constrained by the roads, hence it leads to high resident connection time between two vehicles that are travelling in the same direction. Therefore, we place a great deal of reliance on this feature to give a higher score to a candidate node which travels in the same direction of the packet carrier node. Furthermore, a mobile vehicle can calculate its relative direction with respect to the packet carrier node when its own and the source node's direction are known. Thus, the bearing angle is needed to provide the same or opposite directionality awareness of neighbour vehicles in the vicinity with respect to the packet carrier node. For example: if vehicle $a$ is moving in $\left\langle d x_{a}, d y_{a}\right\rangle$ direction and vehicle $b$ is moving in $\left\langle d x_{b}, d y_{b}\right\rangle$ direction, we can calculate the bearing angle $(\sigma)$ between a candidate node and a packet carrier node as follows:

$\cos \sigma=\frac{d x_{a} \cdot\left(d x_{b}\right)+d y_{a} \cdot\left(d y_{b}\right)}{\left(\sqrt{d x_{a}^{2}+d y_{a}^{2}}\right) \cdot\left(\sqrt{d x_{b}^{2}+d y_{b}^{2}}\right)}$

We now define the score function that gives optimal tradeoff between power signal strength and the relative direction, which is given by:

$g\left(P_{i}, D_{i}\right)=A \times P_{i}^{\gamma_{1}} \times D_{i}^{\gamma_{2}}+B_{\max }$
Where $\gamma_{1}$ and $\gamma_{2}$ are weights for $P$ and $D$ routing metrics respectively. The variable $B_{\max }$ is the maximum time delay after receiving the RTS frame, and $A$ is defined as follows:

$A=\frac{-B_{\max }}{P_{\max }^{\gamma_{1}} \times D_{\max }^{\gamma_{2}}}$

To evaluate equation ??, finding the maximum range of the variables $P$ and $D$ is essential. As determined in section ??, the maximum value of $D$ and $P$ are equal to 1 and $-20 \mathrm{dBm}$ respectively. The value of $A$ can be determined, $A=\frac{-0.8}{0.01^{0.07} \times 1^{0.4}}$ which yields -1.1043 , hence the reply timer becomes $0.0683 \mathrm{~ms}$. Fig. ?? shows the correlation between $P, D$ and $t_{i}$. We observe that, a candidate node has low reply timer if it has good channel quality (strong signal strength) and travels with the same direction of the packet carrier node.

\subsection{IB Protocol Packet Forwarding Example}

In this section, we illustrate the basic operation of the IB geographic forwarding protocol. Note that the main purpose of IB protocol is to forward packets at the intersections and between them without utilizing the periodic hello broadcast message. In the following scenario, we assume that enough vehicles exist at the intersections and between them.

Fig. ?? illustrates how IB protocol works. Now, the packet carrier node $S$, which is at the intersection, needs to send packets to the best preferable candidate node $(A, B, C, F, G, H, I)$, that is, forward it to the destination $D$. First it broadcasts the RTS frame to all nodes within its radio range, which carries the source direction and location as well as the destination location and duration of the communication session. The candidate nodes $R 2$ hear the RTS frame, make sure that the source is between intersections, trigger the score function to determine its reply timer, and the node with the shortest reply timer issues a first reply with CTS frame. In this vehicular scenario, the relay node $(A)$ has the best $D$ and $P$. Therefore, $R 2$ sends back the CTS frame to the source node. When the neighbour nodes hear the CTS frame, they will exit the contention phase and do not send any frame to the source node until $A$ sends an ACK frame to the source node. This vehicular scenario demonstrates the importance of packet forwarding based on the relative direction $D$ between mobile nodes and power signal strength of the RTS frame.

After the best relay node has been selected between intersections, the vehicle $B$ will initiate geographical packet forwarding at the intersection. As noted earlier, the candidate node triggers the score function to calculate its reply timer based on the forwarding progress $G F$, direction $D$ and power signal strength $P$. In consideration of this vehicular 


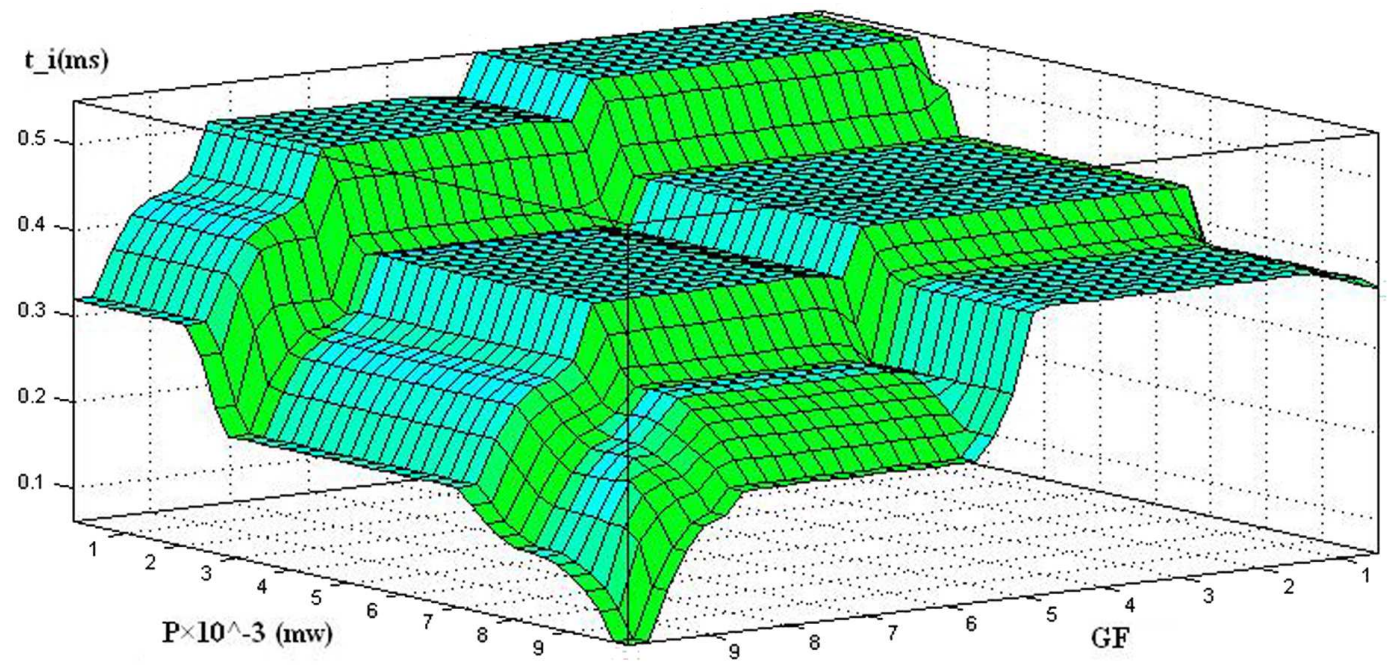

Fig. 5: Correlation between $G F_{i}, P_{i}$ and $t_{i}$ variables $\left(\beta_{1}=0.5, \beta_{2}=0.2, \beta_{3}=0.01\right)$.

scenario, $C$ elects itself as a next hop packet forwarder at the intersection.

\section{Performance Evaluation}

This section presents the evaluation of the IB geographical forwarding protocol in urban vehicular environments. We have simulated the proposed protocol using the packet level simulator JIST/SWANS [?]. It is designed based on the OSI seven layer network communication architecture. The simulation scenario is $3968 \times 1251 \mathrm{~m}$ area that was configured with JIST/SWANS, and the selected area contains 370 road segments with 124 intersections (Fig. ??). We used the open source STreet RAndom Way point mobility model (STRAW) [?] to simulate the movement of vehi- cles. STRAW has an efficient car following trajectory, lane changing model and real-time traffic controller. In STRAW, the generated vehicles are distributed regularly in the urban streets, and they pause for a period of time at the intersections. Moreover, Fig. ?? illustrates the actual map of the Chicago city.

In addition, at the physical layer, the shadowing channel model has been used to characterize the wireless channel [?]. In the simulation, the value of the path loss exponent $\mathrm{n}=2.8$ and the reference distance $d_{0}=0.4$ are used for the shadowing model [?]. Furthermore, we set the radio communication range at 250 meters. In the simulation area, the traffic density of vehicles is varied from 100 nodes to 400 nodes, and they move along the roads with an average speed ranging from 30 to $60 \mathrm{~km} /$ hour. Moreover, the variant of

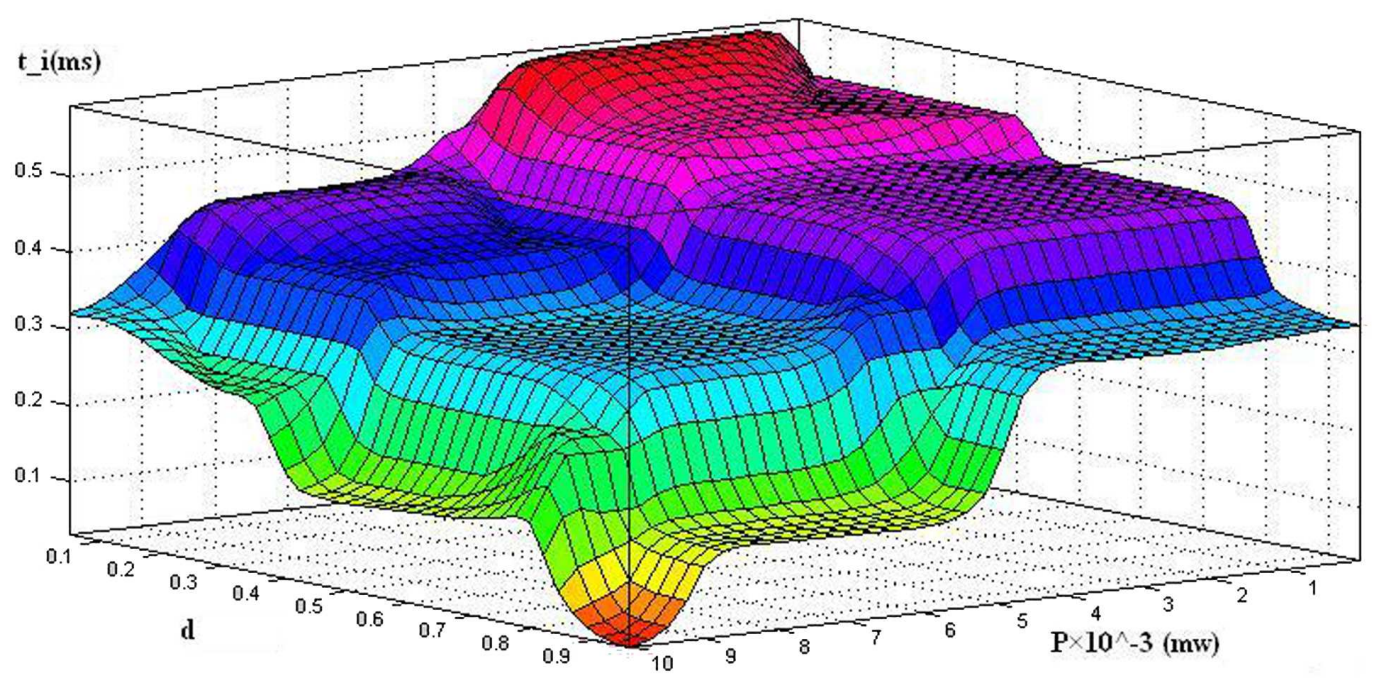

Fig. 6: Correlation between input and output variables $\left(\gamma_{1}=0.07\right.$ and $\left.\gamma_{2}=0.4\right)$. 


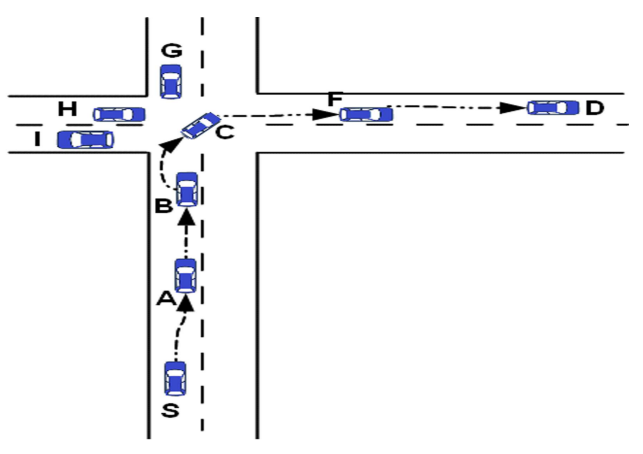

Fig. 7: The illustration of packet forwarding of IB protocol. At the intersection, the receiver $C$ elect itself as a next relay hop, whereas between intersections the node $A, B a n d F$ wins the contention phase.

Table 1: Simulation parameters

\begin{tabular}{|l|l|}
\hline Parameters & Value \\
\hline Simulation time & $350 \mathrm{~s}$ \\
\hline Simulation area & $3968 \mathrm{~m} \times 1251 \mathrm{~m}$ \\
\hline Mobility model & STRAW \\
\hline Traffic Density & $100-400$ nodes \\
\hline Vehicle velocity & $30-60 \mathrm{~km} / \mathrm{hr}$ \\
\hline Transmission range & $250 \mathrm{~m}$ \\
\hline Maximum packet generation rate & 6 packet/second \\
\hline Maximum number of source nodes & 10 \\
\hline Channel bandwidth & $3 \mathrm{Mbps}$ \\
\hline MAC protocol & IEEE $802.11 \mathrm{~b} \mathrm{DCF}$ \\
\hline Data packet size & 512 bytes \\
\hline Weighting factors $\left(\beta_{1}, \beta_{2}, \beta_{3}, \gamma_{1}, \gamma_{2}\right)$ & $(0.01,0.01,0.01,0.3,0.3)$ \\
\hline
\end{tabular}

IEEE 802.11b DCF standard, based upon proposed protocol, is used to model MAC layer [?], [?]. The simulation key parameters are summarized in Table ??. The selection of these simulation parameters is based on the studies $[?, ?, ?, ?]$. This is because these studies were based on the realistic measurements between nearby vehicles. Further, the total simulation time is 350 seconds. We set the settling time to 25 seconds at the beginning of simulation to remove the effect of transient behaviour on the results. The total simulation time also included 25 seconds of stop sending packets from the end of the simulation. It is worth mentioning that each point in the performance figures exemplifies the average of 20 simulation runs.

Accuracy of simulation results are significantly reflect credibility of the data from a specific measurement. Validation is used to evaluate the performance gain which is obtained from the proposed solution. More precisely, statistical significance test, namely Analysis Of Variance (ANOVA-single factor) was calculated to verify the measured data form a specific protocol. ANOVA is a statistical analysis model which is used to partition the variance of a particular variable into components which are attributable to different sources of variation.
We have compared the performance of the IB protocol with the state of the arts geographical routing (GPCR [?] and [?] protocols. We now briefly review the basic operation of these routing protocols: GPCR is a geographical routing protocol that forwards packets to a neighbour node which has the closest distance to the destination (greedy mode of packet forwarding). In the perimeter mode, a node forwards packets to the next neighbour node by applying right hand rule. In addition, GPCR assumes that the road traffic is the planar graph, which utilizes the concept of junction nodes to control the next road segments that packets should follow; CBF uses the distributed timer-based mechanism for the data packet forwarding decision. This random timer mechanism is set when the relay nodes receive the RTS frame and check if they are closer to the destination than the packet carrier node. The contention between relay nodes will end as one of them responds the source by sending CTS frame (Which is a contention winner and selected as the next hop).

The proposed geographical forwarding protocol are compared with routing protocols based on the following evaluation metrics:

(i) Packet Delivery Ratio (PDR): measures the fraction of data packets that are successfully received by the destination to those generated by traffic source.

(ii) End to end delay: is the total time required by all the packets to travel from the source to the destination. The packet delay obtained in the simulation is the sum of sending buffer, medium access (packets delay due to interface queue), re-transmission, relay election and propagation delay.

(iii) Hop count: is the average number of relay nodes that forward data packets to the destination.

In the performance evaluation, we conducted different experiments to study the effect of various parameters on the proposed protocol and the representative of the standard routing protocols.

\subsection{Impact of the Weighting Factors $\beta_{1}, \beta_{2}, \beta_{3}, \gamma_{1}, \gamma_{2}$}

In this section, we conducted experiments to analyze the sensitivity of the weighting factors of the proposed protocol to determine a good balance between the routing metrics. Moreover, the performance of the IB protocol has been simulated for different values of the weighting factors. Fig. ?? and Fig. ?? shows the measured PDR and average packet delay versus the packet sending rate (Constant Bit Rate/Source) for various representative values of the weighting factors.

Initially, as the value of standard deviation and source packet traffic increases, the PDR and average delay remain stable. After the packet generation rate reaches about 32 


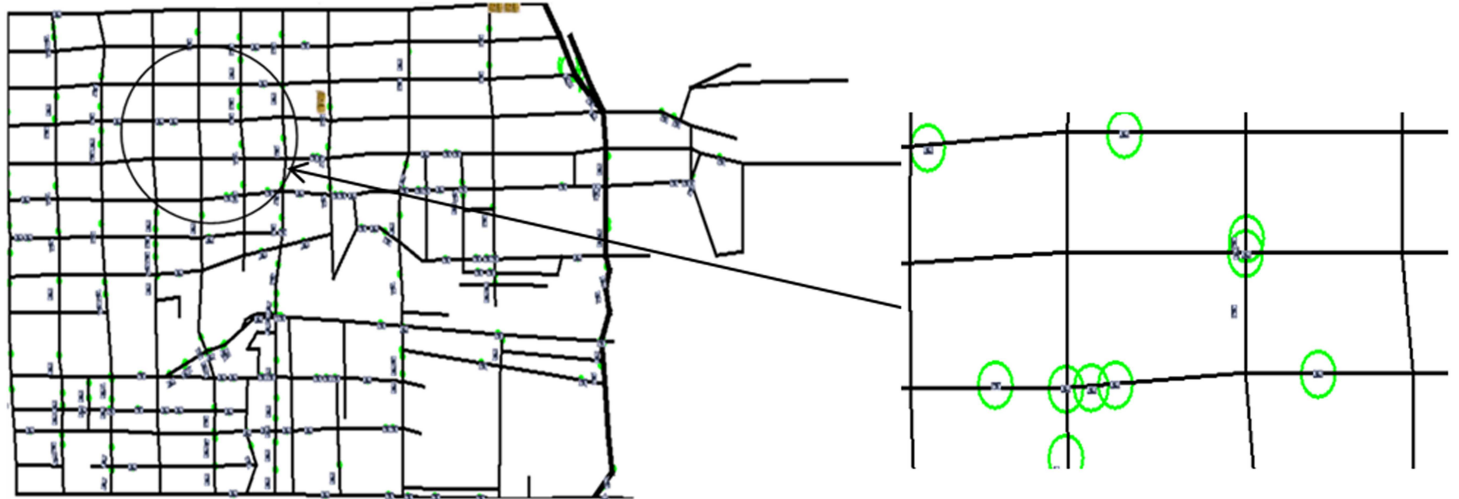

Fig. 8: A snapshot of Chicago city environment during simulation

kbps, we observe upward transition of the packet delay to $420 \mathrm{~ms}$ and decrease of the PDR to $75 \%$ when $\beta_{1}, \beta_{2}, \beta_{3}, \gamma_{1}, \gamma_{2}$ are set to $0.01,0.01,0.01,0.3,0.3$ respectively. We believe that this is because the network reaches its peak saturation throughput (which is the maximum limit of capacity that the network can carry in stable condition) at $32 \mathrm{kbps}$. Afterwards, packet loss occurs due to higher network load. MAC layer tries to compensate for these packet losses at the cost of average packet delay and PDR.

The analysis shows that when the weighting values of $\beta_{1}, \beta_{2}$ and $\beta_{3}$ are equal to each other as well as $\gamma_{1}$ is equal to $\gamma_{2}$, more data packets are successfully delivered with lower average packet delay. We coin the reasons why the values of $\beta_{1}, \beta_{2}, \beta_{3}, \gamma_{1}, \gamma_{2}$ are set to $0.01,0.01,0.01,0.3,0.3$ respec- tively, and offers better performance. First, since the packet $\gamma_{2}$ forwarding based on a single metric lead to sub-optimal, the IB protocol that favours (by using weights) only $G F$, $D$ or $P$ routing metrics does not show good performance. For instance, at the intersection, the IB protocol favours directionality which does not offer optimal performance. This is because the $G F$ routing metric is also important in order to find the shortest path to the destination as well as the $P$ metric has an important role for good quality link selection. Furthermore, if the weights $\left(\gamma_{1}, \gamma_{2}\right)=(0,0.3)$, it means the IB protocol favours signal strength $P$, which finally leads to sub-optimality due to the possibility of selecting unstable (low resident connection time) routes between intersections. Second, the weighting factors together have an impor-

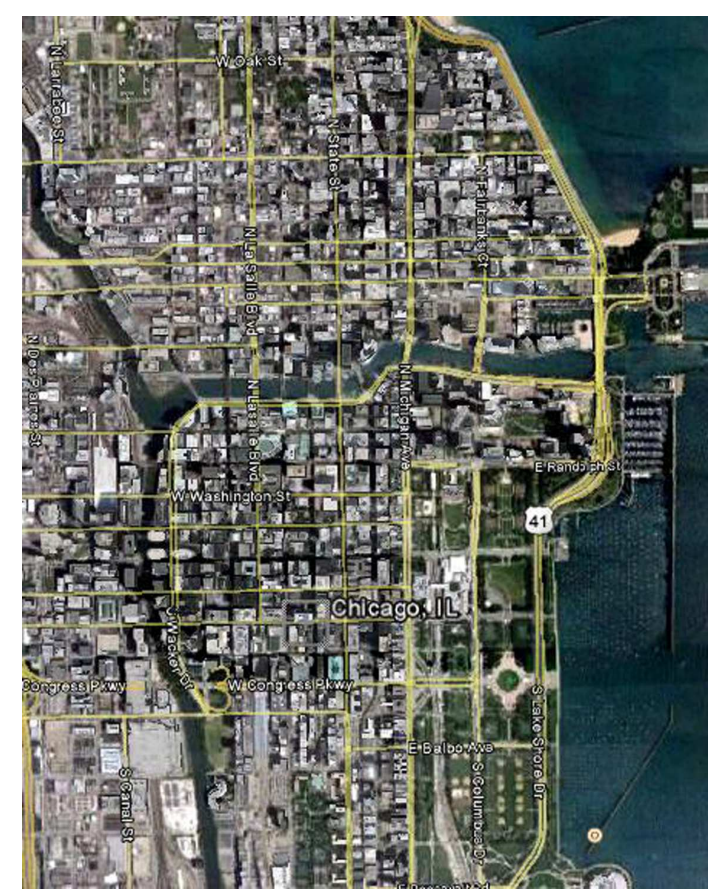

Fig. 9: Map of the region of Chicago city used in the simulation scenario 


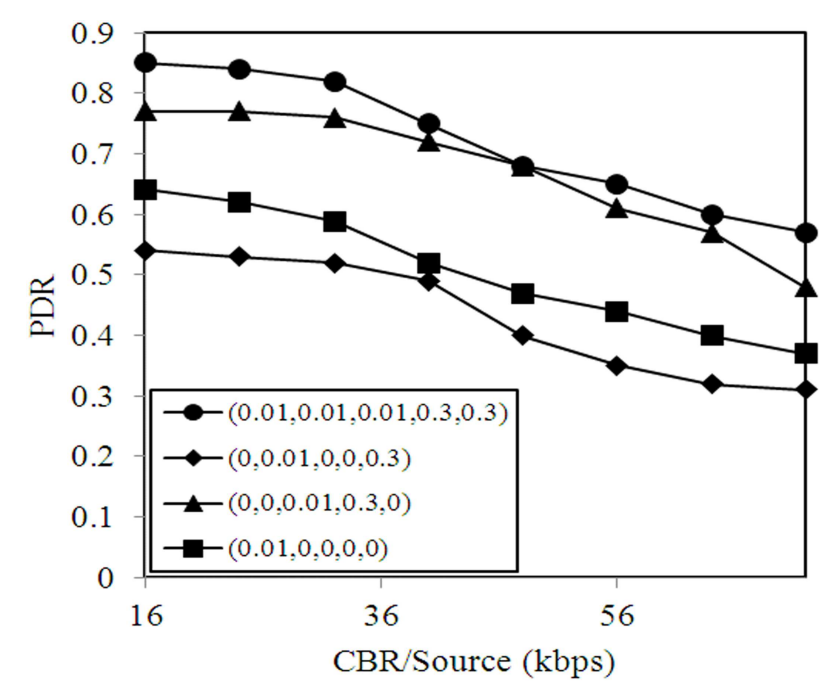

(a) Packet delivery ratio variation with packet sending rate for different weighting factors.

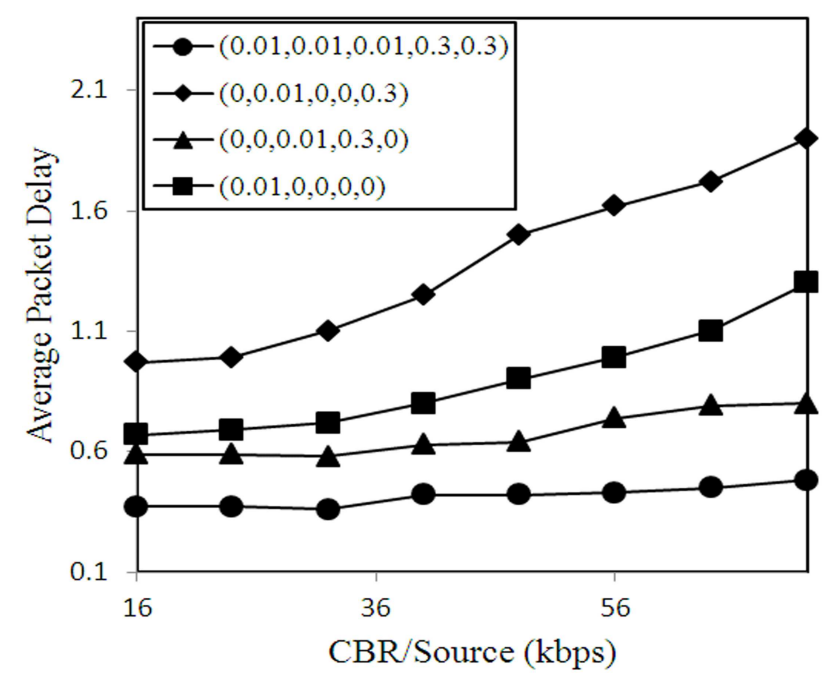

(b) Average packet delay with packet sending rate for different weighting factors.

Fig. 10: Illustration of the effect of different weighting factors on the IB protocol performance.

tant impact in the duration of each election round. Thus, the selected weighting factors attain optimal reply timer of the contention phase between candidate nodes.

\subsection{Impact of Node speed}

This study is performed with a traffic density of 300 nodes with 10 of them acting as a source. To investigate the effect of speed on the performance of the proposed protocol, we run the experiments with varying the mobile speed from 30 $\mathrm{km} /$ hour to $60 \mathrm{~km} /$ hour. The simulated beacon interval is 0.5 $\mathrm{s}$ for the studied (GPCR) protocol.
Fig. ?? shows the packet delivery ratio of the IB, GPCR and $\mathrm{CBF}$ protocols with respect to vehicle speed. A prompt result of this performance evaluation is that an increase in vehicle speed leads to a low successful packet delivery ratio for all protocols. In more detail, as can be seen, the IB geographical forwarding protocol performs better as compared to the other routing protocols. The reasons are that the proposed protocol removes the beacon messages to update the neighbour information, which leads to less bandwidth consumption in the network and the required memory to store neighbour information. As a consequence, the percentage of the link utilization will increase for data packet transfers. Furthermore, the multi-metric based next forwarder election favours more stable and reliable links as well as forwarding progress toward the destination. On the contrary, we observe that the CBF protocol is always lags behind the IB protocol. This is not surprise since CBF protocol only uses greediness factor as a routing metric to forward data packets in the such unreliable and unstable vehicular scenario. Consequently, the trend of CBF drops to $78.8 \%$ at a speed of 60 $\mathrm{km} /$ hour.

ANOVA single factor has been used to compare the means of the proposed IB protocol with the existing protocols. The result indicates that the IB protocol has the lowest variance compared with the state of the arts. The variance of IB, GPCR and CBF are $0.001329,0.008859$ and 0.002004 respectively for PDR with $F$ value of 44.301 and $P$ less than $1 \%$ level of significance. These results suggest that the IB protocol has lower variance than other two protocols. The implication is that the proposed IB protocol may be more efficient in increasing PDR in the urban vehicular scenario than the other two methods as it shown in Fig. ??. Thus, the applied ANOVA single factor validation method is significantly reflect credibility of the variance of data from specific measurement of the proposed protocol.

In contrast to beaconless forwarding protocols, in GPCR protocol, the packet carrier node needs to know the position information of all direct neighbours. This information is obtained through periodic beacon messages sent out by each direct neighbour node. The high mobility of vehicles leads to the staleness of neighbourhood information. As a result, the trend of GPCR protocol acutely drops to $57.5 \%$ at a speed of $60 \mathrm{~km} /$ hour.

In addition, even though each direct neighbour node utilizes its own accurate location information, the IB and $\mathrm{CBF}$ protocols suffer slightly when mobility increases to $60 \mathrm{~km} /$ hour. We believe that this is because the elected direct neighbour node will exit the radio range before receiving the actual data packets or sends back the CTS frame to the source.

In Fig. ??, we show the effect of increasing vehicle speed on average packet delay. The proposed protocol has the smallest average delay among the protocols studied. In IB proto- 


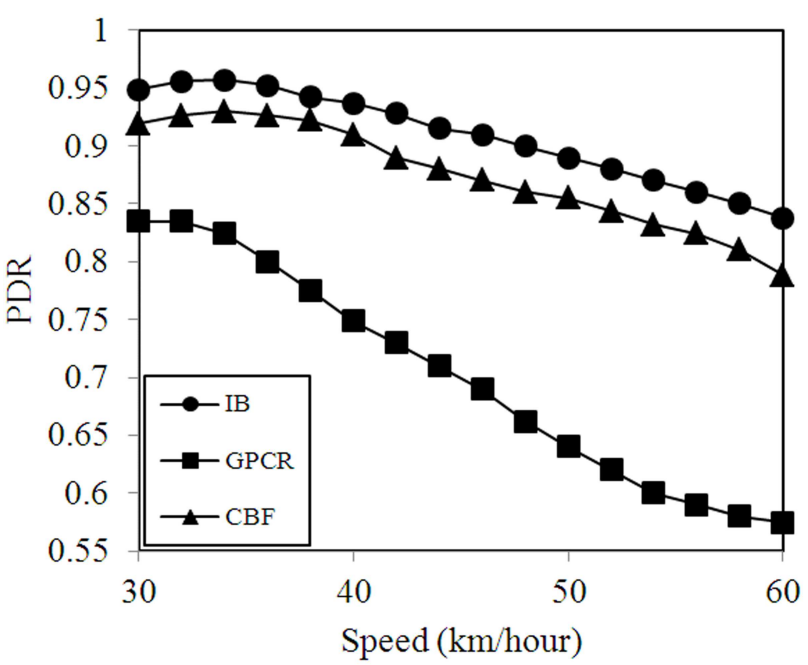

(a) Packet delivery ratio.

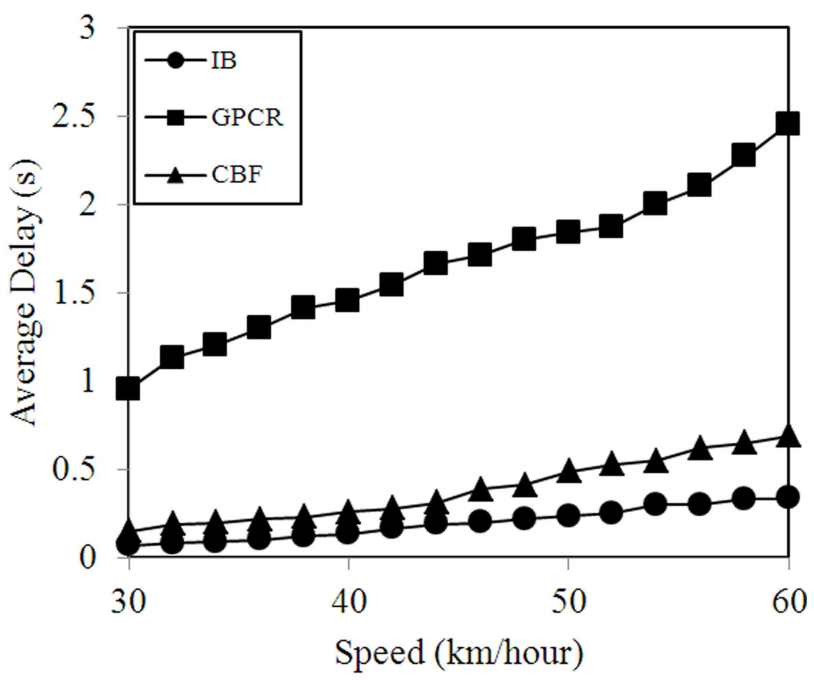

(b) Average packet delay.

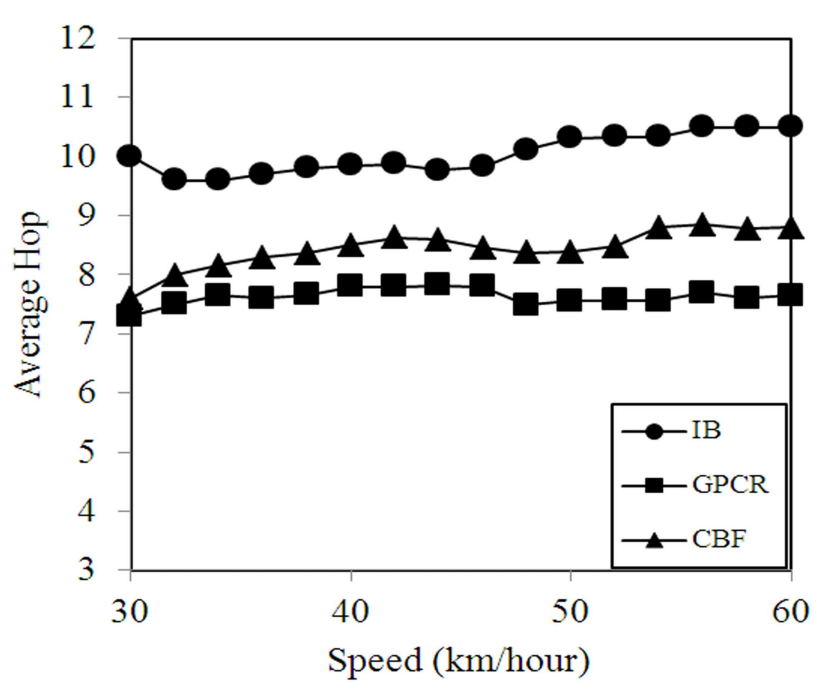

(c) Average path length.

Fig. 11: Effect of varying vehicle speed on the performance of IB, GPCR and CBF protocols. col, the route is determined based on the modified RTS/CTS frames handshaking, and this mechanism is more effective in reducing the traffic load on the MAC layer. This leads to improved delays, because fewer retransmissions and exponential backoffs happen in the MAC layer. Contrarily, in GPCR, the average delay increases drastically with higher mobility. This is because the number of MAC layer retransmissions increases.

In addition, we observe that the CBF protocol suffers in terms of average delay as compared to the IB protocol. This can be attributed to the fact that the relay nodes in CBF contend to access the channel based on greediness factor; that is, a relay node elect itself as a next packet forwarder when it has the shortest distance to the destination. Only considering greediness factor for packet forwarding leads to RTS frame, CTS frame or data packet losses in unreliable wireless channels between vehicles. As a result, MAC layer tries to perform redundant retransmissions to compensate these RTS frame, CTS frame or data packet losses. With these packet retransmissions, the $\mathrm{CBF}$ protocol is susceptible to higher end-to-end delay. As can be seen in Fig. ?? the delay trend of CBF increases to $691 \mathrm{~ms}$ at a speed of $60 \mathrm{~km} / \mathrm{hour}$.

The comparison between IB protocol and the state of the arts in Fig. ?? indicates that our proposed protocol has slightly longer average path length than the other protocols. The reason is that, unlike GPCR and CBF, IB protocol explores the paths to the destination by considering link reliability (considering power strength), link stability (considering direction) and forwarding progress toward the destination (greedy forwarding). Expectedly, the routing protocols should perform better for shorter path lengths. However, the results do not support this hypothesis, because selecting better en-route nodes leads to better performance. For instance, the IB protocol has a longer path length, but it performs better than other studied protocols.

\subsection{Impact of Traffic Density}

In this study, we conducted experiments to understand the effects of a variable number of vehicles on the performance of proposed and existing solutions. The experiments involved setting the vehicle speed at $45 \mathrm{~km} /$ hour and the number of source nodes at 10 . We ran the simulation with different number of nodes ranging from 100 to 400 .

The results of this experiment is plotted in Fig. ??. In Fig. ??, the trend of the average delivery ratio is plotted with the different number of vehicles. As expected, the trend of protocols show that the successful packet delivery ratio consistently increased as the number of vehicles increases. This is not surprising since the probability of connectivity is increased with the increasing vehicular traffic density. In more detail, when node density is sufficiently high (300 nodes or 


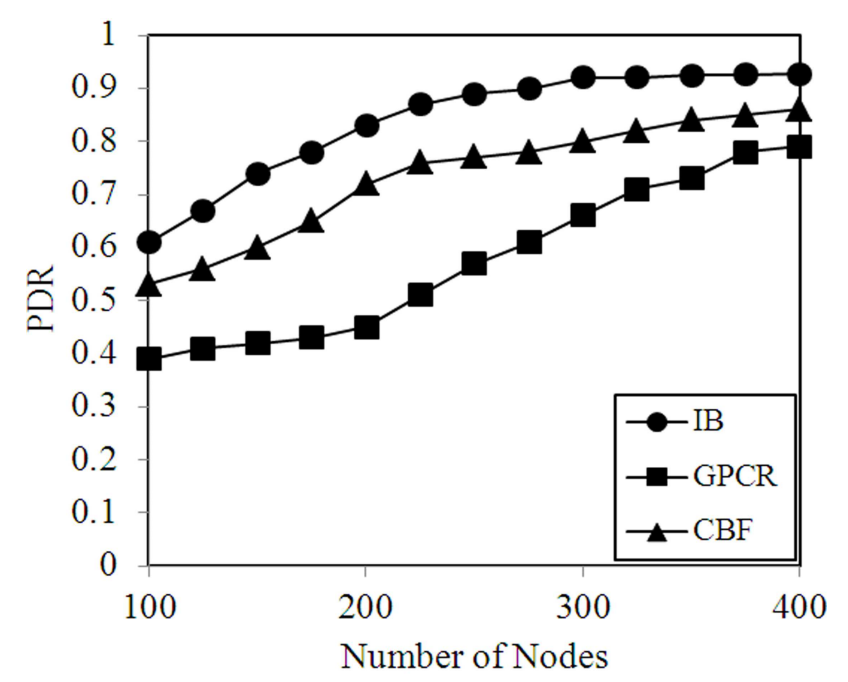

(a) Packet delivery ratio.

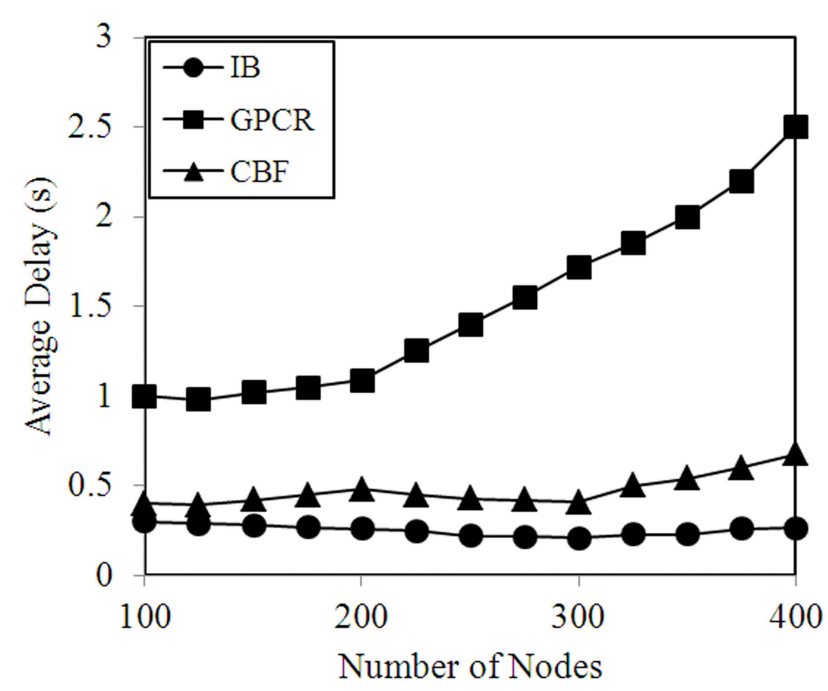

(b) Average packet delay.

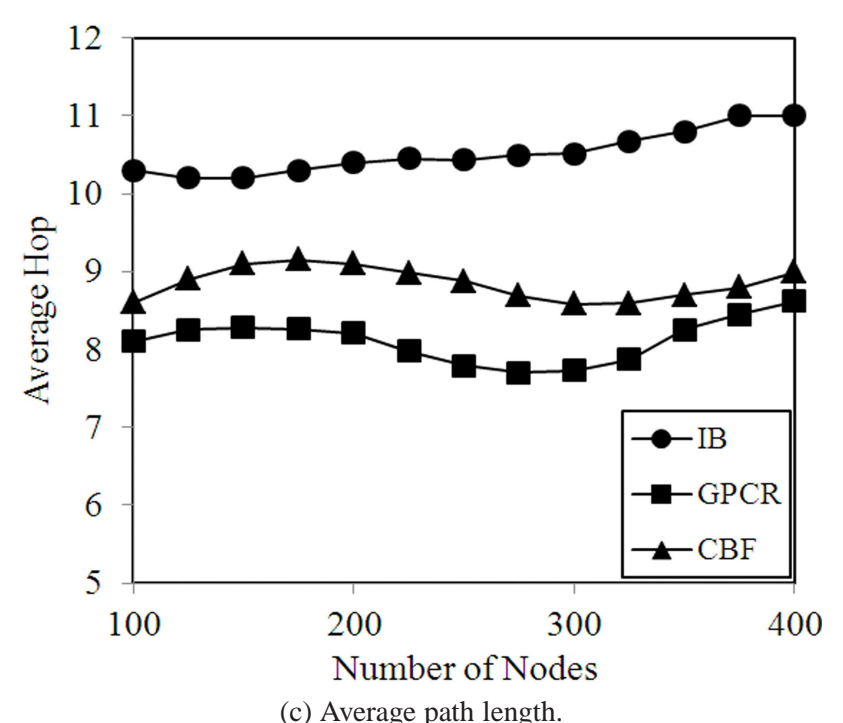

Fig. 12: Effect of varying vehicular traffic density on the performance of IB, GPCR and CBF protocols. more), the IB protocol's trend becomes flat. This is because the RTS/CTS handshaking procedure increases the probability that a packet collision will occur as the packet is routed towards the destination. The GPCR protocol, on the other hand, greedily forwards data packets toward the destination. The link between the packet carrier node and the selected next hop will be very weak (move out the radio range). This case leads to fewer packets delivered to the specified destination.

The CBF protocol uses relay node self election mechanism to greedily forward data packets toward the destination. In greedy packet forwarding, the probability of link failure increases due to high signal attenuation of unreliable wireless channels. As a result, the network performance is degraded due to high packet loss. Due to this case, the IB protocol performs better as compared to the CBF protocol.

Another interesting metric is the average packet delay, which is depicted in Fig. ??. We notice that the average packet delay for IB protocol consistently decreases until the number of nodes becomes 300 , then rises slightly to $265 \mathrm{~ms}$ at 400 nodes. The reason is that low traffic density in the network increases the likelihood that the network will be dis-connected during the forwarding process, whereas the high traffic density leads to packet collision and duplication. Consequently, in both cases, the average packet delay slightly increases. The average packet delay of CBF, on the other hand, increases to $675 \mathrm{~ms}$ as number of nodes reach $\mathbf{4 0 0}$. The responsibility of CBF's high latency lies in the increasing number of MAC layer retransmissions.

In GPCR, The average packet delay steeply increases with traffic density. There are two reasons for this: First, when the number of nodes increases, the time to determine next packet forwarder (which is close to the destination) also increases. Second, unlike IB protocol, GPCR does not favour link reliability and stability.

Fig. ?? shows the average path length variation with traffic density. Comparing the hop count incurred by IB protocol with those obtained by the state of the arts, we notice that the average path length of IB protocol is slightly longer than that of GPCR and CBF. However, our proposed protocol offers better performance in terms of successful delivery ratios and average end-to-end delay. The reason for this effect lies again in the favouring link reliability and stability in addition to the forwarding progress.

\subsection{Impact of Radio Obstacles on the Simulation Results}

In this study, we conducted experiments to understand the effects of radio obstacles on the performance of proposed and existing solutions. The experiments involved setting the vehicle speed at $45 \mathrm{~km} /$ hour, the traffic density at 250 nodes 
and the number of source nodes at 10 . We ran the simulation with different packet generation rate ranging from 16 to 72 kbps.

The static obstacles (buildings) in the urban environment is simulated in the following model. The roadmap of the urban area is represented as graphs where streets are straight road segment. In this city map representation, two vehicles in the same road segment are considered to be in line-ofsight due to non-existence of buildings to interfere with the radio signal. This can be determined by obtaining the street number in road segment file for each vehicle. If two vehicles are in the same street (visible to each other), they can communicate with each other. If this case is not satisfied, i.e., there will be a building or an open area between two vehicles, the log-normal shadowing model in JIST/SWANs is modified in order to add the attenuation value to the signal attenuation between transmitter and receiver.

Fig. ?? shows that the proposed IB protocol performs better with performance increases of up to $10 \%$ compared with GPCR and $6.25 \%$ compared with CBF. We observe that the successful delivery ratio decreases as the packet generation rate increases. But, the trend of the routing solutions is not sharp, which means the routing solutions can maintain the traffic load, as compared with the simulation results without radio obstacles. This is not surprise since the the presence of radio obstacles in the vehicular scenario reduces the percentage of contention on the MAC sub-layer. On the other hand, as the value of source packet traffic increases, the PDR decreases accordingly. This performance hit of the routing solutions is due to increased error rate of the wireless link between the packet carrier node and its neighbours.

In Figure ??, the average packet delay is plotted with respect to packet generation rate for different routing protocols. When the inter-packet arrival time is large, the average packet delay increases for IB,GPCR and CBF Protocols. But, this ascending of delay is different for each protocol. For IB protocol, the trend starts at about $625 \mathrm{~ms}$ then fluctuates gradually until it reaches $753 \mathrm{~ms}$ at $72 \mathrm{kbps}$. The reason of this gradual increase of delay is that, when obstacles are present in the city map, the contention in the wireless network will be low. But, the increase of delay is due to the unreliability of wireless channels between vehicles.

\section{Conclusions}

In this article, we proposed an Intelligent Beaconless (IB) geographical forwarding protocol to optimally route data packets towards the destination. As discussed in detail in this article, the proposed protocol consists of two modes of packet forwarding: at the intersection and between them.

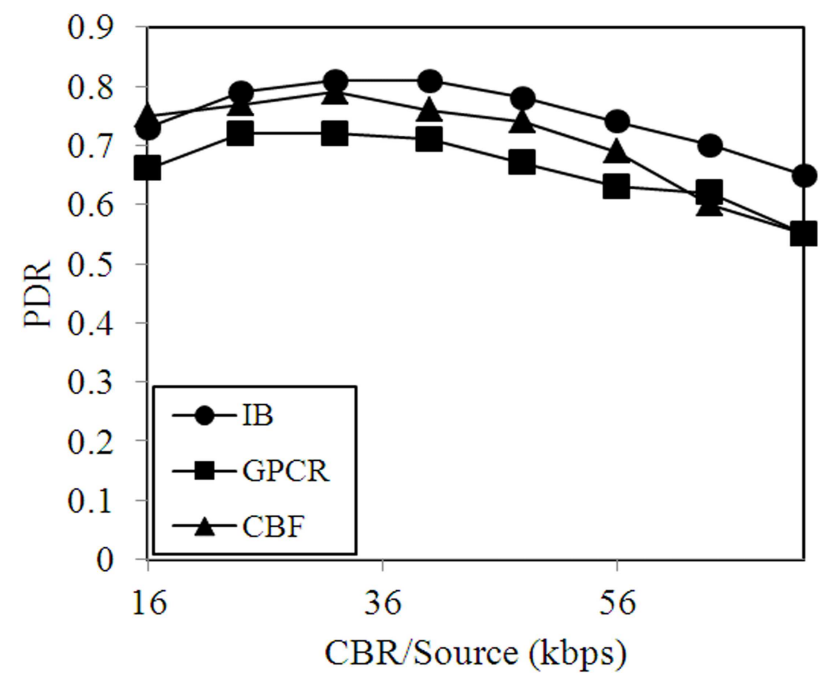

(a) Packet delivery ratio.

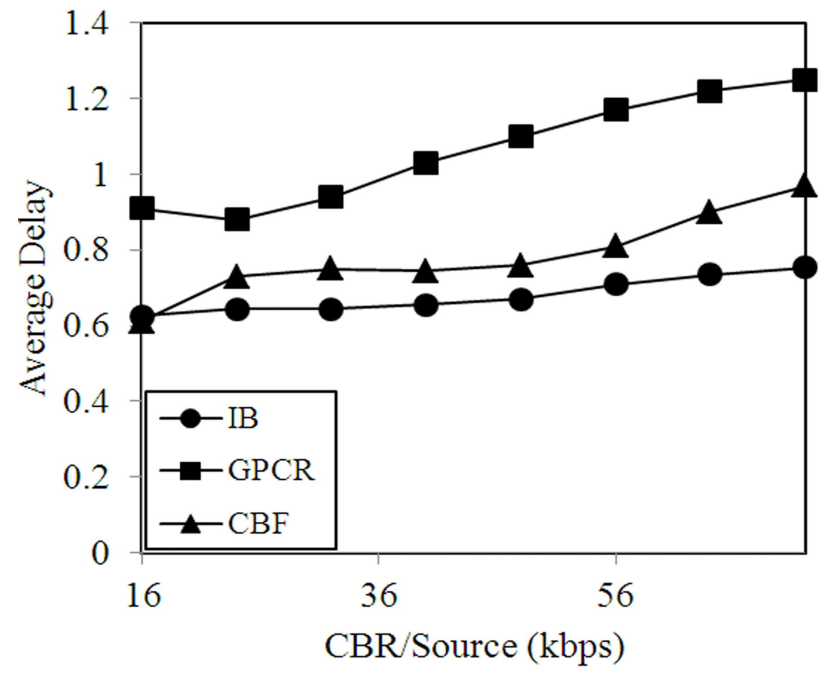

(b) Average packet delay.

Fig. 13: Effect of radio obstacle on the performance of IB, GPCR and CBF protocols.

Both modes of packet forwarding, which are modelled analytically, rely on distributed next hop self election based on the modified 802.11 RTS/CTS frames. In addition, the IB protocol forwards data packets along the city streets by considering the real traffic on the roads and realistic wireless channels. Simulation results show that, compared to the representatives of geographical (GPCR and $\mathrm{CBF}$ ) routing protocol, the IB protocol performs the best in terms of successful packet delivery ratio and average packet delay. The future work will consider the implementation of the proposed IB protocol with IEEE 802.11p. In addition, we are currently working to model static (e.g., Buildings) and moving (e.g., bus, fire track) obstacles by designing a new attenuation and visibility schemes (both schemes have significant effect on wireless signal propagation model). 
Acknowledgements The authors wish to thank the anonymous reviewers for their constructive comments in improving the quality of this manuscript.

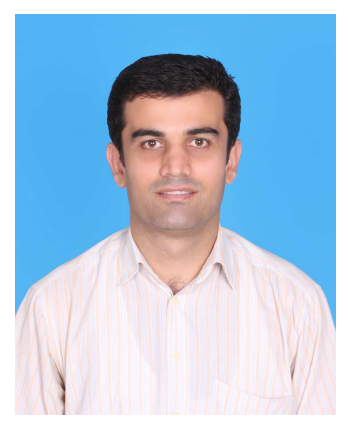

Kayhan Zrar Ghafoor received the BSc degree in Electrical Engineering from Salahaddin University, the MSc degree in Remote Weather Monitoring from Koya University and the PhD degree in Wireless Networks from University Technology Malaysia in 2003, 2006, and 2011 , respectively. He is serving as a member of Editorial Board of IJCSIS, IJAIT, IJACSA, JCIT and IJRRAN. He is also serving as an associate editor of IJNPA and IJANC. Kayhan is currently working as a technical committee of GreeNets 2012, ICNCS 2012, SMARTGREENS, TACPS 2012 and IEEE International Workshop on Smart Communication Protocols and Algorithms. He has been as a reviewer for 10 international journals and 2 international conferences. Kayhan has awarded UTM International Doctoral Fellowship (IDF) and Kurdistan Regional Government (KRG) scholarship (Ahmad Ismail Foundation). His current research interests include routing over Vehicular Ad Hoc Networks and Tactical Wireless Networks, as well as Artificial Intelligence and network coding applications. He is a member of IEEE Communications Society and International Association of Engineers (IAENG).

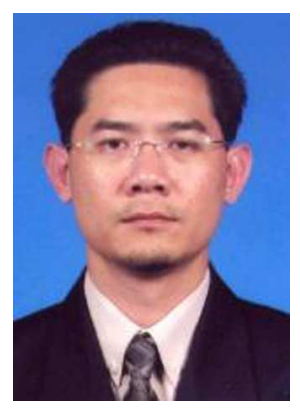

Kamalrulnizam Abu Bakar obtained his PhD degree from Aston University (Birmingham, UK) in 2004. Currently, he is associate professor in Computer Science at Universiti Teknologi Malaysia (Malaysia) and member of the "pervasive Computing" research group. $\mathrm{He}$ involves in several research projects and is the referee for many scientific journals and conferences. His specialization includes mobile and wireless computing, information security and grid computing.

Jaime Lloret (M07 SM10), re-

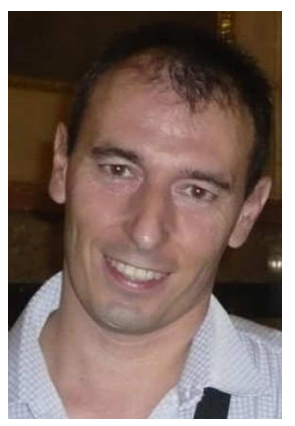

Associate Professor in the Polytechnic University of Valencia and he is the research line coordinator of the "communications and remote sensing" of the Integrated Management Coastal Research Institute. He is coordinating the "Active and collaborative techniques and use of technologic resources in the education (EITACURTE)" Innovation Group. He is the director of the University Expert Certificate Redes y Comunicaciones de Ordenadores and of the University Expert Certificate Tecnologas Web y Comercio Electrnico. He is currently the Cognitive Networks Technical Committee (IEEE Communications Society) Vice-chair for the Europe/Africa Region. He has more than 155 research papers published in national and international conferences, international journals (most of them with Impact Factor in Journal Citation Report), and books. He has 11 educational books and more than 55 papers published in international conferences, journals and books of education. He has been the co-editor of 15 conference proceedings and guest editor of several international books and journals. He is editor-in-chief of the international journal "Networks Protocols and Algorithms", editor-in-chief of the international Journal "Advances in Network and Communications", IARIA Journals Board Chair (8 Journals) and he is associate editor of several international journals. He has been involved in more than 150 Program committees of international conferences and in several organization and steering committees until 2011. He has been the general chair of SENSORCOMM 2007, UBICOMM 2008, ICNS 2009 and ICWMC 2010 and co-chairman of ICAS 2009 and INTERNET 2010. He is the co-chairman of IEEE MASS 2011 and SCPA 2011. He is IEEE Senior Member and IARIA Fellow Member.

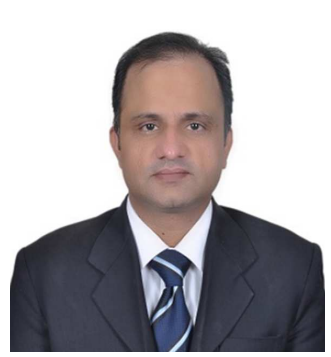

Rashid Hafeez Khokhar did his M.S. in Statistics from University of the Punjab, Lahore, Pakistan. He received his M.S. in Computer Science from Preston University, Pakistan. Dr.Rashid also received his second M.S. in Computer Science by research from Universiti Teknologi Malaysia, Malaysia. He earned $\mathrm{PhD}$ in Computer Science at

Universiti Teknologi Malaysia. He is currently working as a senior lecturer in Faculty of Computer Science and Information Technology, University of Malaya, Malaysia. His areas of interest are geographical routing and realistic propagation modelling in Vehicular Ad hoc Network. 


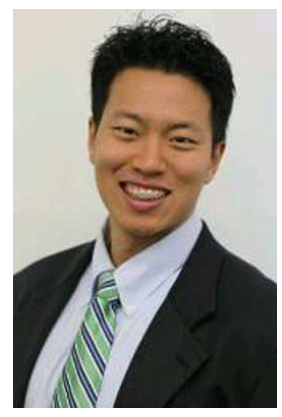

Kevin C. Lee received his Ph.D. in the Computer Science Department at the University of California, Los Angeles in 2010. He received a B.S. in Computer Science Engineering and B.A. in Mathematics at the University of Pennsylvania in 2002. He also received an M.S. in Computer Science at the Carnegie Mellon University in 2004. Kevin has published several papers in the fields of vehicular ad hoc networks ranging from developing and running a peer-to-peer application on the real vehicular test-bed, designing an optimized geographic routing protocol in urban scenarios, developing a light-weight loop-free geographic routing protocol, and proposing link-state routing based on density. He has also written technical reports in network process migration in the area of network system programming and in classification in the area of artificial intelligence. His current research interests include vehicular ad hoc network routing, theoretical analysis of computer networks, WiMax networks, QoS, and mobile applications for vehicular ad hoc networks. 